\title{
The Hyperbilirubinemia and Potential Predictors Influence on Long-Term Outcomes in Sepsis: A Population-Based Propensity Score-Matched Study
}

OPEN ACCESS

Edited by:

Penglin Ma

Guiqian International General

Hospital, China

Reviewed by:

Zhongheng Zhang,

Sir Run Run Shaw Hospital, China

Yong Ming Yao,

First Affiliated Hospital of Chinese PLA

General Hospital, China

${ }^{*}$ Correspondence:

Desheng Qi

qidesheng17@csu.edu.cn

Zhonghua Hu

huzhonghua@csu.edu.cn

Lina Zhang

zln7095@163.com

†These authors have contributed equally to this work

Specialty section

This article was submitted to

Intensive Care Medicine and

Anesthesiology,

a section of the journal

Frontiers in Medicine

Received: 24 May 2021

Accepted: 16 August 2021

Published: 17 September 2021

Citation:

Peng M, Deng F, Qi D, Hu Z and

Zhang $L$ (2021) The

Hyperbilirubinemia and Potential

Predictors Influence on Long-Term

Outcomes in Sepsis: A

Population-Based Propensity

Score-Matched Study.

Front. Med. 8:713917.

doi: 10.3389/fmed.2021.713917
Milin Peng ${ }^{1,2}$, Fuxing Deng ${ }^{1,2}$, Desheng $\mathrm{Qi}^{2,3 * t}$, Zhonghua Hu ${ }^{1,2,4 * t}$ and Lina Zhang ${ }^{1,2 * t}$

${ }^{1}$ Department of Critical Care Medicine, Xiangya Hospital, Central South University, Changsha, China, ${ }^{2}$ National Clinical Research Center for Geriatric Disorders, Xiangya Hospital, Central South University, Changsha, China, ${ }^{3}$ Department of Emergency, Xiangya Hospital, Central South University, Changsha, China, ${ }^{4}$ Hunan Key Laboratory of Molecular Precision Medicine, Xiangya Hospital, Institute of Molecular Precision Medicine, Central South University, Changsha, China

Objective: Although hyperbilirubinemia has been associated with mortality in patients who are critically ill, yet no clinical studies dissect the effect of dynamic change of hyperbilirubinemia on long-term septic prognosis. The study aims to investigate the specific stages of hyperbilirubinemia and potential risk factors on long-term outcomes in patients with sepsis.

Methods: In this retrospective observational cohort study, patients with sepsis, without previous chronic liver diseases, were identified from the Medical Information Mart for the Intensive Care III MIMIC-III database. We used propensity scores (PS) to adjust the baseline differences in septic patients with hyperbilirubinemia or not. The multivariate Cox was employed to investigate the predictors that influence a clinical outcome in sepsis.

Results: Of 2,784 patients with sepsis, hyperbilirubinemia occurred in 544 patients (19.5\%). After PS matching, a survival curve demonstrated that patients with sepsis with the new onset of total bilirubin (TBIL) levels more than or equal to $5 \mathrm{mg} / \mathrm{dl}$ survived at significantly lower rates than those with TBIL levels $<5 \mathrm{mg} / \mathrm{dl}$. Multivariate Cox hazard analysis showed that patients with TBIL at more than or equal to $5 \mathrm{mg} / \mathrm{dl}$ during sepsis exhibit 1.608 times (95\% Cl: 1.228-2.106) higher risk of 1-year mortality than those with TBIL levels $<5 \mathrm{mg} / \mathrm{dl}$. Also, age above 65 years old, preexisting malignancy, a respiratory rate above 30 beats/min at admission, serum parameters levels within 24-h admission, containing international normalized ratio (INR) above 1.5, platelet $<50^{*} 10^{\wedge} \mathrm{g} / \mathrm{L}$, lactate above $4 \mathrm{mmol} / \mathrm{L}$, and bicarbonate $<22$ or above $29 \mathrm{mmol} / \mathrm{L}$ are the independent risk factors for long-term mortality of patients with sepsis.

Conclusions: After PS matching, serum TBIL levels at more than or equal to $5 \mathrm{mg} / \mathrm{dl}$ during hospitality are associated with increased long-term mortality for patients with sepsis. This study may provide clinicians with some cutoff values for early intervention, which may improve the prognosis of patients with sepsis.

Keywords: sepsis, liver, risk factors, mortality, hyperbilirubinemia 


\section{INTRODUCTION}

Sepsis is defined as a life-threatening acute organ dysfunction secondary to infection. A high incidence rate and high mortality of sepsis make it one of the leading causes of death as a global health priority $(1,2)$. Notably, sepsis-induced organ dysfunction is an important predictor for poor prognosis (35). The liver plays a central role in homeostasis, immune surveillance, inflammation, and bacterial clearance $(6,7)$. A large body of evidence has suggested that the liver is the main target of sepsis and decompensation of liver function can trigger overwhelming inflammation, immune response, and organ damage in sepsis $(8,9)$. However, despite extensively studied lung, kidney, and heart injury in the course of sepsis, the question of whether dysfunction of the liver is associated with mortality or a poor outcome in sepsis remains unresolved. Hepatic dysfunction and hyperbilirubinemia commonly occur in patients who are critically ill with an incidence rate of $40 \%$ and up to $20 \%$ in patients with bacterial infection (10).

Hyperbilirubinemia may result from bacterial products or as a consequence of the response of the host to infection. The etiology of hyperbilirubinemia in patients who are critically ill is multifactorial, probably cholestasis or sclerosing cholangitis caused by circulating endotoxins, inflammation, hypoxia hepatitis, lower liver perfusion and ischemia, genetic and metabolic variations, and so on (11-16). Hyperbilirubinemia has been shown to represent an important marker of mortality and poor outcomes for patients who are critically ill $(17,18)$. Patients with a preexisting liver deficit, like cirrhosis, have a worse outcome of sepsis than ones without liver dysfunction due to impaired immunity (19). The underlying mechanism may relate to the reversal of bilirubin transport from intrahepatic toward the circulation, which is beneficial for relieving the highenergy burden for hepatocytes and serves as a metabolic and inflammatory stress response as well (20). Hence, elevated serum bilirubin might indicate the impairment of energy consumption due to liver injury, and total serum bilirubin level has been widely recognized as a powerful maker for assessing hepatic function compared with other serum activities on laboratory tests.

Thus far, several studies have demonstrated the potential effect of liver dysfunction on short-term mortality of patients with sepsis by using different definitions of hepatic dysfunction (21-23). Whereas, it is still unclear of the long-term impact of dynamic change of hyperbilirubinemia in patients with sepsis. The appendant result derived from PROWESS-SHOCK (Prospective Recombinant Human Activated Protein C Worldwide Evaluation in Severe Sepsis and Septic Shock) trial showed that liver dysfunction is associated with 180-day

\footnotetext{
Abbreviations: MIMIC, medical information mart for intensive care; STROBE, STrengthening the Reporting of OBservational studies in Epidemiology; ICD9, International Classification of Diseases, 9th revision; IRB, institutional review board; SOFA, sequential organ failure assessment; TBIL, total bilirubin; CHF, congestive heart failure; AFIB, atrial fibrillation; CAD, coronary artery disease; COPD, chronic obstructive pulmonary disease; ALT, alanine aminotransaminase; AST, aspartate aminotransferase; $\mathrm{CI}$, confidence interval; HR, hazard ratio; OR, odds ratio; $\mathrm{pCO}_{2}$, partial pressure of $\mathrm{CO}_{2}$; INR, international normalized ratio; BAIBF, bile acid-independent bile flow; USA, United States.
}

mortality in patients with septic shock by using criteria of serum bilirubin $\geq 20 \mathrm{mmol} / \mathrm{L}$ (24). However, as hyperbilirubinemia is multifactorial, there is no large study dissecting the effect of liver dysfunction on long-term mortality of more than 180 days in patients with general sepsis. Therefore, we performed a large cohort study to analyze the dynamic change of hyperbilirubinemia and its influence on outcomes in patients with sepsis by using an openly available US-based critical care database named Medical Information Mart for Intensive Care (MIMIC)-III v 1.4, which includes 52,963 ICU admissions. Our results revealed a significant association between total bilirubin (TBIL) levels with long-term mortality in patients with sepsis.

\section{METHODS}

\section{Study Population}

We conducted the cohort study according to the STrengthening the Reporting of OBservational studies in Epidemiology (STROBE) statement by using the MIMIC-III database, a large, integrated, de-identified, open-free, comprehensive clinical dataset, comprised of all the patients admitted to the ICUs Beth Israel Deaconess Medical Center in Boston, MA, from June 2001 to October 2012. For all of the data that are deidentified, patient consent or ethics approval will not be needed. Demographics records, laboratory results, radiology examinations, diagnosis, clinical treatment parameters, and dates of death were also concluded. The diagnosed diseases by the physician were according to the International Classification of Diseases, 9th revision (ICD-9) on patient discharge. Since the study was an analysis of a third-party anonymized publicly available database with preexisting institutional review board (IRB) approval, approval from our institution was exempted.

Sepsis was defined according to Sepsis-3 criteria: the suspected infection and Sequential Organ Failure Assessment (SOFA) score was of 2 points or more $(25,26)$. Hyperbilirubinemia was diagnosed in patients with the new onset of serum TBIL at more than or equal to $2 \mathrm{mg} / \mathrm{dl}$ during the hospitalization. The included criteria were as follows: age $\geq 18$ years old; patients without previous chronic liver diseases according to the recorded ICD9 codes, including liver cirrhosis; at ICU admission more than $24 \mathrm{~h}$; missing data $<50 \%$. The excluded criteria were preexisting bilirubin at more than or equal to $2 \mathrm{mg} / \mathrm{dl}$ before admission, and previous chronic liver diseases like chronic hepatitis, acute-onchronic liver failure, cirrhosis, liver cancer, hepatobiliary ductrelated tumors, or acute liver conditions like drug or toxininduced hepatitis. After being included, all the patients with sepsis were divided into a hyperbilirubinemia group (serum TBIL during hospitalization at more than or equal to $2 \mathrm{mg} / \mathrm{dl}$ ) and a non-hyperbilirubinemia group (serum TBIL $<2 \mathrm{mg} / \mathrm{dl}$ ).

\section{Measures and Variable Definition}

For the patients in the study, we retrieved demographic and admission information from the database during the first $24 \mathrm{~h}$ of ICU admission, including age, gender, ethnicity (White, Hispanic, Black, or other), weight, time of admission or discharge, the severity of illness parameters: the SOFA score (clarified into four different strata: 2-4, 5-9, more than or 
equal to 10 scores) and the Elixhauser comorbidity score, and vital signs: heart rate (clarified into two different strata: $<100$ beats/min, more than or equal to 100 beats/min), and respiratory rate (clarified into two different strata: $<30$ beats/min, more than or equal to 30 beats/min). In addition, we routinely collected laboratory parameters within the first $24 \mathrm{~h}$ of ICU admission, including maximum levels of white blood cell count (WBC); levels of hemoglobin $(\mathrm{g} / \mathrm{dl})$; hematocrit; platelet levels (stratified into five different scales: more than or equal to $250^{*} 10^{\wedge} 9 / \mathrm{L}$, at $150-249^{*} 10^{\wedge} 9 / \mathrm{L}$, at $100-149^{*} 10^{\wedge} 9 / \mathrm{L}$, at $50-99^{*} 10^{\wedge} 9 / \mathrm{L}$, and $<50^{*} 10^{\wedge} 9 / \mathrm{L}$ ); serum potassium/sodium/chloride levels; serum bicarbonate levels (stratified into three different scales: $<22$ $\mathrm{mmol} / \mathrm{L}$, at $22-29 \mathrm{mmol} / \mathrm{L}$, and more than $29 \mathrm{mmol} / \mathrm{L}$ ); serum blood urea nitrogen (BUN)/creatinine; serum lactate levels (stratified into four different scales: at $0-2 \mathrm{mmol} / \mathrm{L}$, at $2.1-$ $4 \mathrm{mmol} / \mathrm{L}$, at $4.1-10 \mathrm{mmol} / \mathrm{L}$, and more than $10 \mathrm{mmol} / \mathrm{L}$ ); international normalized ratio (INR, stratified into two different scales: $\leq 1.5$, more than 1.5); arterial blood gas with $\mathrm{PH}$, partial pressure of oxygen ( $\mathrm{pO} 2)$, partial pressure of carbon dioxide (pCO2); alanine transaminase (ALT)/aspartate transaminase (AST). We also collected parameters in the course of sepsis: use of mechanical ventilation, use of vasopressor agents, and use of sedative drugs. By collecting in 24, 48, and 72 h, 7 days, and the day of discharge after ICU admission, serum levels of TBIL were classified into four different scales: at $0-1.9,2-4.9,5-10 \mathrm{mg} / \mathrm{dl}$, and more than $10 \mathrm{mg} / \mathrm{dl}$.

We also included preexisting medical comorbidities according to the recorded ICD-9 codes, including congestive heart failure $(\mathrm{CHF})$, renal disease, atrial fibrillation (AFIB), coronary artery disease (CAD), chronic obstructive pulmonary disease (COPD), stroke, and malignant tumor. The Kidney Disease: Improving Global Outcomes (KDIGO) clinical practice guidelines were used to define acute kidney injury (AKI). We conducted the followup at day 30 (30 days), 90 days, 180 days, and 1 year from the database.

\section{Statistical Analysis}

Statistical analysis was performed using SPSS 23 (SPSS, Inc., Chicago, IL). The parameters with missing data of more than $50 \%$ were excluded from our study. The mean-value imputation algorithm was selected to substitute missing values. Baseline characteristics and clinical parameters after ICU admission between the hyperbilirubinemia group and the control group were compared. All continuous variables were expressed as means (SD) or medians (interquartile range, IQR) by using either Student $t$-test or Mann-Whitney $\mathrm{U}$ test as appropriate. Categorical variables were compared by the chi-square test or Fisher's exact test. The survival curves for patients with sepsis were plotted using the Kaplan-Meier method, and differences between the curves were assessed using the log-rank test. To identify the association between the dynamic change and the levels of hyperbilirubinemia and a long-term outcome in sepsis, Cox regression analysis was used. Univariate Cox proportional hazards regression of clinical parameters was performed to identify potential predictors. Hazard ratios (HRs) were calculated with $95 \%$ CIs as an estimate of the risk associated with a particular variable. To determine independent predictors of the composite end points, variables in univariate Cox analysis with $p<0.1$ were entered into multivariate Cox proportional hazards regression, and the predictors were performed with a likelihood ratio-forward selection.

To account for selection bias and potential confounding factors between groups in comparison of an outcome, we used the propensity score (PS) matching (1:1) to balances covariates for those who had hyperbilirubinemia and those who had not (532 pairs). A multivariable logistic regression model with confounding baseline characteristics was used to calculate the PS for each patient as the predicted probability of the hyperbilirubinemia group. The following variables were adjusted (Table 1): age; sex; race/ethnicity; preexisting medical conditions: CHF, AFIB, CAD, COPD, stroke, malignant tumor, and chronic renal disease; Elixhauser comorbidity score; respiratory rate; and biochemical parameters. One-to-one nearest-neighbor matching without replacement with a caliper width of.1 was conducted. We evaluated the balance test after matching, with no significant difference with chi-square $\left(X^{2}=16.468, P=0.870\right)$, which demonstrated a good balance following PS matching between the hyperbilirubinemia and non-hyperbilirubinemia groups.

After matching, survival curves were computed and plotted using the Kaplan-Meier method. Univariate and multivariate Cox proportional hazards regression models were constructed to explore the independent risk factors of influence on long-term outcomes in sepsis. A $P<0.05$ (two-sided) was considered significant.

\section{RESULTS}

Of the 52,963 ICU admissions from the MIMIC-III database, 5,784 patients meet the definition of sepsis. About 3,310 participants were identified in our analysis according to the inclusion criteria; afterward, patients with preexisting liver disease $(n=526)$ were excluded from our study. Finally, $544(19.5 \%)$ patients developed hyperbilirubinemia, while the remaining 2,240 (80.5\%) patients did not (Figure 1).

\section{Patient Characteristics Before Matching}

Table 1 shows the notable differences in baseline characteristics between the hyperbilirubinemia and non-hyperbilirubinemia groups of sepsis before PS matching. The results showed that men were prone to develop hyperbilirubinemia in the course of sepsis (58.3 vs. $53.6 \%$; $P=0.049)$. The hyperbilirubinemia group had higher prevalence of preexisting medical comorbidities, including AFIB (32.2 vs. 26.4\%; $P=0.009)$, and malignancy (27.9 vs. $21.3 \% ; P=0.001$ ); lower prevalence of COPD (9.7 vs. $14.9 \%$; $P=0.001)$, stroke (3.5 vs. $8.7 \% ; p<0.001$ ), and renal disease (15.4 vs. $21.8 \% ; P=0.001)$ than the non-hyperbilirubinemia group (Table 1).

There was also a significantly higher severity of illness in the hyperbilirubinemia group than the non-hyperbilirubinemia group, with higher rates of the SOFA score at more than 4 (83.9 vs. 56.1\%; $p<0.001$ ), higher levels of Elixhauser comorbidity index [5. (10) vs. 4. (9)], and higher frequency of the respiratory rate at more than or equal to 30 beats/min ( 45.6 vs. $38.4 \%$; $P=0.002$; Table 1). At $24 \mathrm{~h}$ after admission to ICU, the 
TABLE 1 | Demographic characteristics between hyperbilirubinemia and non-hyperbilirubinemia groups before propensity score matching.

\begin{tabular}{|c|c|c|c|}
\hline Parameters & \multicolumn{2}{|c|}{ Full cohort } & $P$ \\
\hline Age, mean (SD), y & $67.19(27)$ & $67.27(26)$ & 0.777 \\
\hline Male, $n(\%)$ & 317 (58.3) & 1200 (53.6) & 0.049 \\
\hline \multicolumn{4}{|l|}{ Race, $n(\%)$} \\
\hline Hispanic & $15(2.8)$ & $67(3.0)$ & 0.888 \\
\hline Black & $42(7.7)$ & $236(10.5)$ & 0.055 \\
\hline Other & 106 (19.5) & 368 (16.4) & 0.098 \\
\hline Weight, mean (SD) & $81.0(25)$ & $80.0(25)$ & 0.292 \\
\hline \multicolumn{4}{|l|}{ Preexisting medical conditions, $n(\%)$} \\
\hline $\mathrm{CHF}$ & $134(24.6)$ & $579(25.8)$ & 0.584 \\
\hline Stroke & $19(3.5)$ & $194(8.7)$ & $<0.001$ \\
\hline Malignancy & $152(27.9)$ & $476(21.3)$ & 0.001 \\
\hline Renal disease & $84(15.4)$ & $489(21.8)$ & 0.001 \\
\hline SOFA score at admission, $n$ (\%) & & & $<0.001$ \\
\hline Score at 2 4 & 88 (16.2) & $982(43.8)^{\star}$ & \\
\hline Score at 5 9 & $274(50.4)$ & $1004(44.8)^{\star}$ & \\
\hline Score at $\geq 10$ & $182(33.5)$ & $254(11.3)^{\star}$ & \\
\hline Elixhauser comorbidity index, mean (SD) & $5.00(10)$ & $4.00(9)$ & $<0.001$ \\
\hline Heart rate $\geq 100$ (beats/min), $n(\%)$ & $362(66.5)$ & $1414(63.1)$ & 0.149 \\
\hline Respiratory rate $\geq 30$ (beats/min), $n(\%)$ & $248(45.6)$ & $860(38.4)$ & 0.002 \\
\hline Platelet at 50 99 & $47(8.6)$ & $93(4.2)^{*}$ & \\
\hline Platelet at $<50$ & $19(3.5)$ & $35(1.6)^{*}$ & \\
\hline Maximum potassium, mean (SD) & $4.50(1.2)$ & $4.50(1.0)$ & 0.734 \\
\hline Maximum sodium, mean (SD) & $140.00(6.0)$ & $141.00(5.0)$ & 0.051 \\
\hline Maximum bicarbonate levels, $n(\%)$ & & & $<0.001$ \\
\hline At $<22$ & $192(35.3)$ & $558(24.9)^{\star}$ & \\
\hline At $22-29$ & $314(57.7)$ & $1398(62.4)^{\star}$ & \\
\hline At $>29$ & $38(7.0)$ & $284(12.7)^{\star}$ & \\
\hline Maximum chloride, mean (SD) & $108.00(8.0)$ & $108.00(8.0)$ & 0.926 \\
\hline Maximum Bun, mean (SD) & $29.00(29.75)$ & $26.00(26.00)$ & 0.060 \\
\hline Maximum lactate levels(mmol/L), $n$ (\%) & & & $<0.001$ \\
\hline Lactate at 0-2 & $190(34.9)$ & $1029(45.9)^{\star}$ & \\
\hline Lactate at $2.1-4$ & $171(31.4)$ & $767(34.2)$ & \\
\hline Lactate at $4.1-10$ & $149(27.4)$ & $392(17.5)^{\star}$ & \\
\hline Lactate at >10 & $34(6.3)$ & $52(2.3)^{\star}$ & \\
\hline Maximum creatinine, mean (SD) & $1.40(1.30)$ & $1.30(1.20)$ & 0.010 \\
\hline Maximum hematocrit, mean (SD) & $35.3(7.85)$ & $35.5(8.60)$ & 0.838 \\
\hline \multicolumn{4}{|l|}{ Maximum INR levels, $n$ (\%) } \\
\hline At $>1.5$ & $288(52.9)$ & $651(29.1)$ & $<0.001$ \\
\hline
\end{tabular}


TABLE 1 | Continued

\begin{tabular}{|c|c|c|c|}
\hline \multirow[t]{2}{*}{ Parameters } & \multicolumn{2}{|c|}{ Full cohort } & \multirow[t]{2}{*}{$\boldsymbol{P}$} \\
\hline & $\begin{array}{l}\text { Hyperbilirubinemia group } \\
\qquad(n=544)\end{array}$ & $\begin{array}{l}\text { No-hyperbilirubinemia group } \\
\qquad(n=2240)\end{array}$ & \\
\hline Maximum $\mathrm{PH}$, mean (SD) & $7.40(0.07)$ & $7.40(0.07)$ & 0.377 \\
\hline Maximum pO2, mean (SD) & $214.0(113)$ & $214.0(142)$ & 0.921 \\
\hline Maximum pCO2, mean (SD) & $47.0(11)$ & $47.0(9)$ & 0.030 \\
\hline Maximum ALT, mean (SD) & $117.00(292.50)$ & $30.00(52.00)$ & $<0.001$ \\
\hline Maximum AST, mean (SD) & $155.00(366.75)$ & $41.00(77.00)$ & $<0.001$ \\
\hline Maximum total bilirubin at $24 \mathrm{~h}$, mean (SD) & $3.30(3.10)$ & $0.50(0.50)$ & $<0.001$ \\
\hline Maximum total bilirubin at $48 \mathrm{~h}$, mean (SD) & $3.50(3.50)$ & $0.60(0.50)$ & $<0.001$ \\
\hline
\end{tabular}

Continuous variables are reported as mean (Standard deviation, SD), and Categorical variables are reported as count (\% of column total).

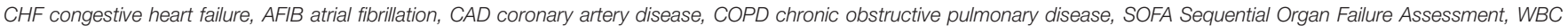
white blood cell, INR International Normalized Ratio, BUN blood urea nitrogen, AST aspartate transaminase, $A L T$ alanine transaminase, $\mathrm{pCO}_{2}$ partial pressure of carbon dioxide, $\mathrm{PO}_{2}$ partial pressure of oxygen.

${ }^{*}$ Represent significant difference with $P<0.05$.

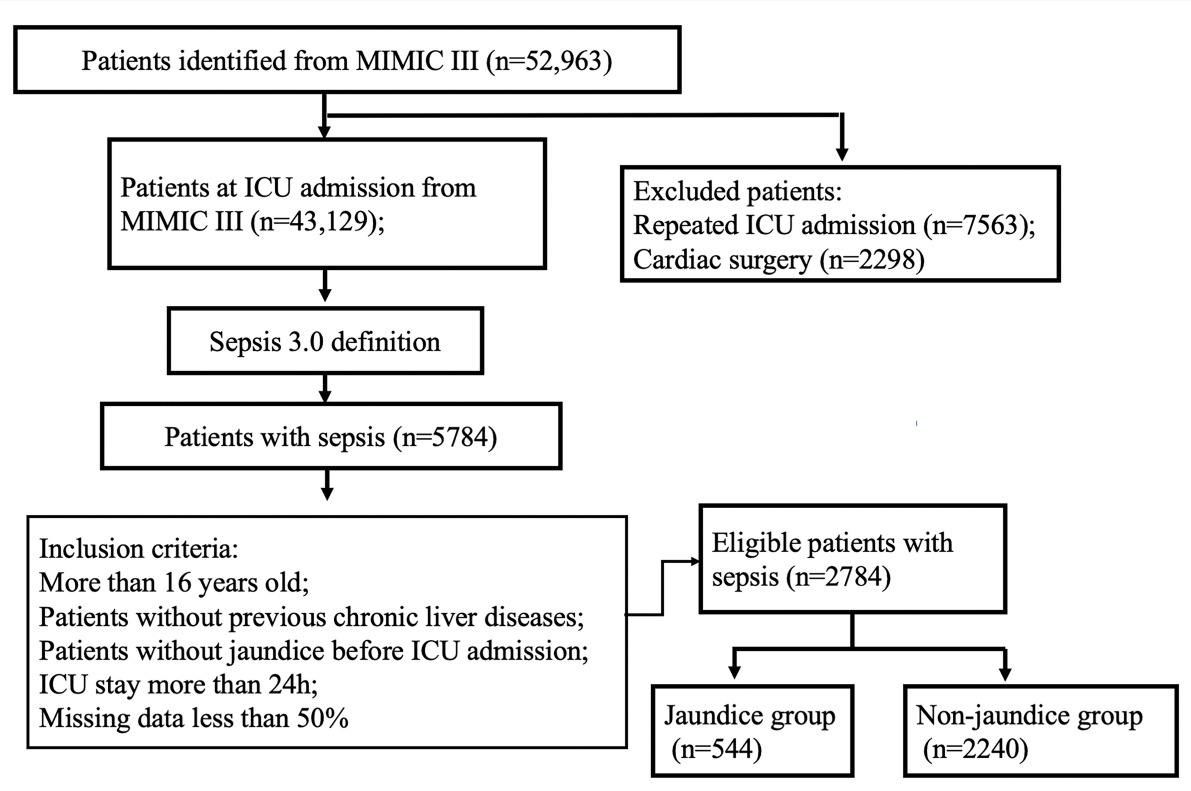

FIGURE 1 | A flow chart of patient selection.

hyperbilirubinemia group had significant higher rates of serum platelet levels at $<150^{*} 10^{\wedge} 9 / \mathrm{L}(30.1$ vs. $17.2 \%$; $p<0.001$ ), serum bicarbonate levels at $<22 \mathrm{mmol} / \mathrm{L}$ ( 35.3 vs. $24.9 \%$; $p<0.001$ ), serum lactate levels at more than $4 \mathrm{mmol} / \mathrm{L}$ (33.7 vs. $29.8 \%$; $p$ $<0.001$ ), and INR levels at more than 1.5 (52.9 vs. $29.1 \%$; $p<$ 0.001 ) when compared with the non-hyperbilirubinemia group (Table 1).

The patients from the non-hyperbilirubinemia group all had TBIL levels below $1.9 \mathrm{mg} / \mathrm{dl}$. By contrast, $62.1 \%$ of patients from the hyperbilirubinemia group had serum TBIL levels at 2-4.9 $\mathrm{mg} / \mathrm{dl}, 25.9 \%$ had TBIL levels at $5-10 \mathrm{mg} / \mathrm{dl}$, and $11.9 \%$ had TBIL levels at more than $10 \mathrm{mg} / \mathrm{dl}$ (Table 2). In addition, the hyperbilirubinemia group had higher rates of vasopressor usage when compared with the non-hyperbilirubinemia group. The overall in-hospital mortality of patients with sepsis was $23.5 \%$. The patients in the hyperbilirubinemia group had significantly higher rates of in-hospital mortality (16.2 vs. $10.7 \%, P=0.002)$, 30 -day mortality (21.7 vs. $16.3 \%, P=0.011)$, 90-day mortality (25.4 vs. $19.5 \%, P=0.010$ ), 180 -day mortality $(27.2$ vs. $22.1 \%$, $P=0.039)$, and 1-year mortality after discharge (29.8 vs. $24.5 \%, P=0.037)$ than in the non-hyperbilirubinemia group, respectively (Table 2). Kaplan-Meier's analysis also showed that the 1-year survival rate was significantly lower in the hyperbilirubinemia group than in the non-hyperbilirubinemia group $(P=0.006)$ before matching (Figure 2A). Moreover, it is of note that TBIL levels more than or equal to $5 \mathrm{mg} / \mathrm{dl}$ at 
TABLE 2 | Clinical outcomes between hyperbilirubinemia and non-hyperbilirubinemia groups before propensity score matching.

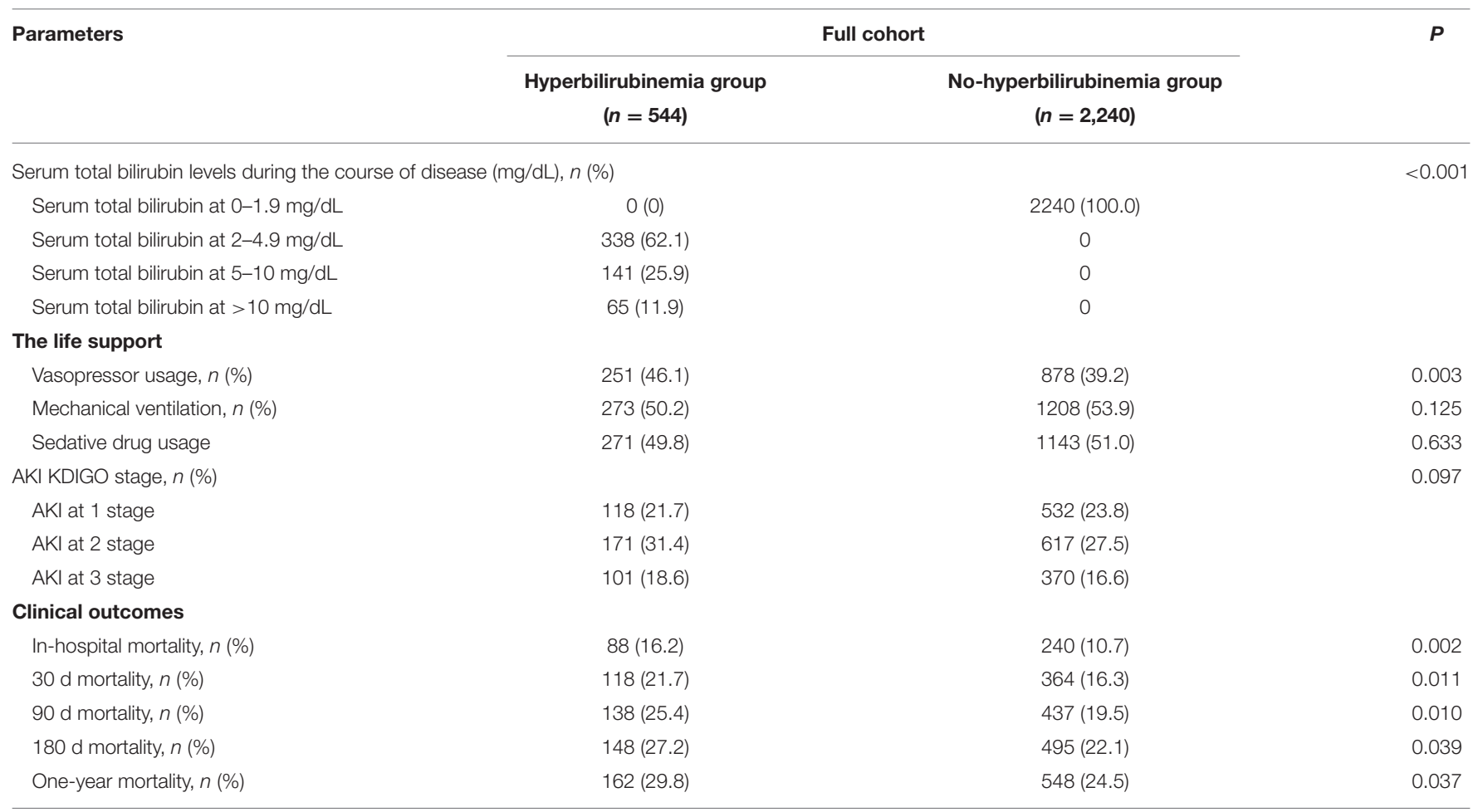

Categorical variables are reported as count (\% of column total).

Abbreviations: AKI acute kidney injury, KDIGO the Kidney Disease: Improving Global Outcomes.

the hospital significantly increases the risk of mortality in sepsis $(p<0.001$, Figure 2B).

\section{Univariate and Multivariate Cox Hazard Analysis of Risk Factors for Mortality in Sepsis Before Matching}

Having identified that several laboratory serum activities, such as serum platelet levels, bicarbonate levels, lactate levels, and INR levels that are significantly associated with hyperbilirubinemia in ICU, we tested whether these serum activities, as well as other clinical outcomes, could be predictors of sepsis prognosis. We first deployed the univariate Cox proportional hazard model to analyze the predictors of mortality in sepsis. We employed a variety of categorical variables with a reference variable: serum platelet levels with platelet levels above or equal to $250^{*} 10^{\wedge} \mathrm{g} / \mathrm{L}$ being a reference variable; serum lactate levels with lactate levels $\leq 2 \mathrm{mmol} / \mathrm{L}$ being a reference variable; serum bicarbonate levels with bicarbonate levels at $22-29 \mathrm{mmol} / \mathrm{L}$ being a reference variable; serum TBIL levels with TBIL levels $<2 \mathrm{mg} / \mathrm{dl}$ being a reference variable; the AKI KDIGO stage without AKI as a reference variable.

Univariate analysis indicated that age at more than 65 years, weight, race in Black, Hispanic, or other, preexisting medical conditions (Malignancy, Stroke, AFIB, and renal disease), Elixhauser comorbidity index, respiratory rate at more than $30 \mathrm{~m}$ beats/min, serum INR levels at more than 1.5 , serum platelet levels at $<50^{*} 10^{\wedge} 9 / \mathrm{L}$, serum lactate levels at more than
$2 \mathrm{mmol} / \mathrm{L}$, serum bicarbonate levels at more than $29 \mathrm{mmol} / \mathrm{L}$, or $<22 \mathrm{mmol} / \mathrm{L}, \mathrm{AKI}$ at two or three stages, mechanical ventilation usage, vasoactive drug usage, and serum TBIL at more than or equal to $5 \mathrm{mg} / \mathrm{dl}$ were the significant risk factors in mortality in sepsis.

To test the prognostic predictors of mortality in sepsis, we performed multivariate analysis using the Cox proportional hazard model for all variables identified as significant by univariate analysis. Following control of confounders and Likelihood ratio (LR) forward elimination, results indicated (Table 3) that TBIL levels at 5-10 $\mathrm{mg} / \mathrm{dl}$ during sepsis have a correlation with 1-year mortality risks [HR 1.396; 95\% CI (1.040-1.875); $P=0.027]$. The finding showed that age above 65 years old [Hazard ratio, HR 2.169; 95\% CI (1.829-2.572); $p<$ 0.001 ], other race [HR 1.391; 95\% CI (1.158-1.671); $p<0.001$ ], preexisting malignancy [HR 1.821; 95\% CI (1.544-2.146); $p<$ 0.001 ], preexisting stroke [HR 1.604; 95\% CI (1.258-2.044); $p<$ 0.001], Elixhauser comorbidity index at admission [HR 1.019; 95\% CI (1.008-1.031); $P=0.001$ ], respiratory rate above 30 beats/min at admission [HR 1.442; 95\% CI (1.242-1.675); $p<$ 0.001 ], serum INR above 1.5 at $24 \mathrm{~h}$ of admission [HR 1.217; $95 \%$ CI (1.038-1.426); $P=0.016$ ], serum platelet $<50^{*} 10^{\wedge} 9 / \mathrm{L}$ at $24 \mathrm{~h}$ of admission [HR 1.442; 95\% CI (1.242-1.675); $p<0.001$ ], serum lactate more than $10 \mathrm{mmol} / \mathrm{L}$ at $24 \mathrm{~h}$ of admission [HR 2.605; 95\% CI (1.880-3.609); $p<0.001]$, serum bicarbonate $<22 \mathrm{mmol} / \mathrm{L}$ [HR 1.375; 95\% CI (1.162-1.626); $p<0.001$ ], or above $29 \mathrm{mmol} / \mathrm{L}$ [HR 1.434; 95\% CI (1.140-1.804); $P=0.002$ ], and mechanical 

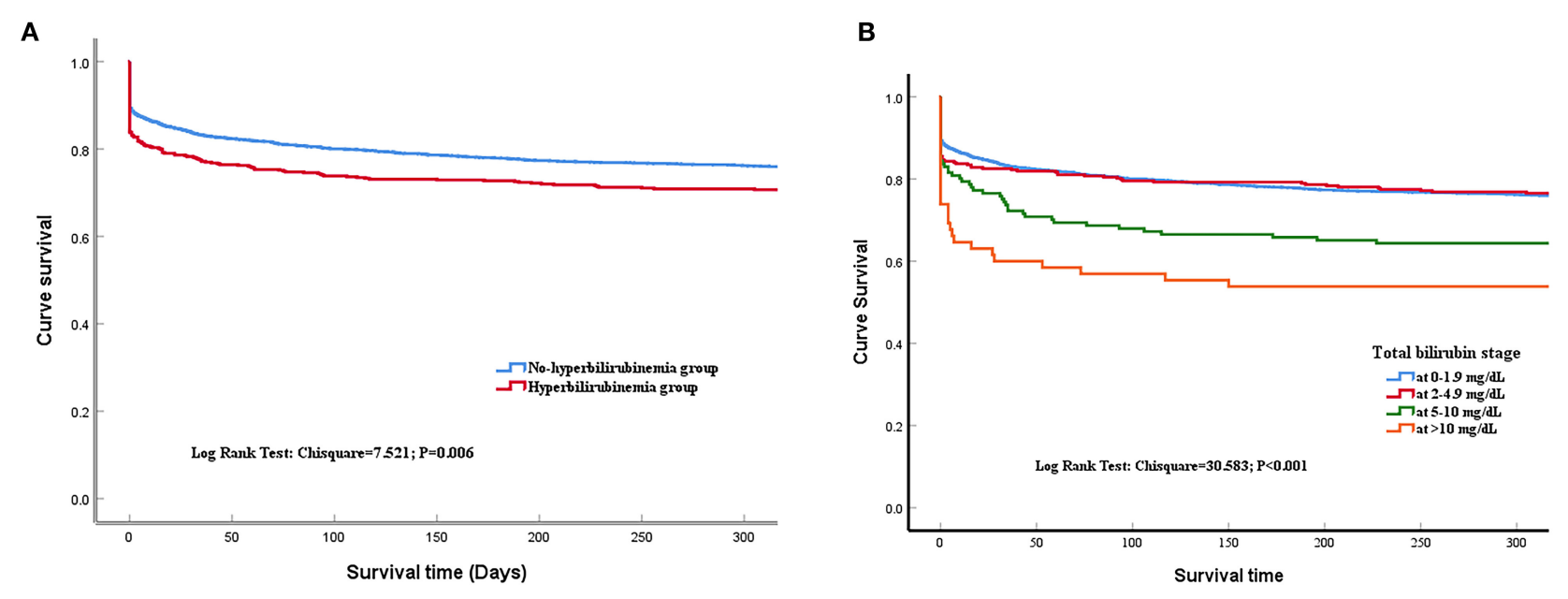

C

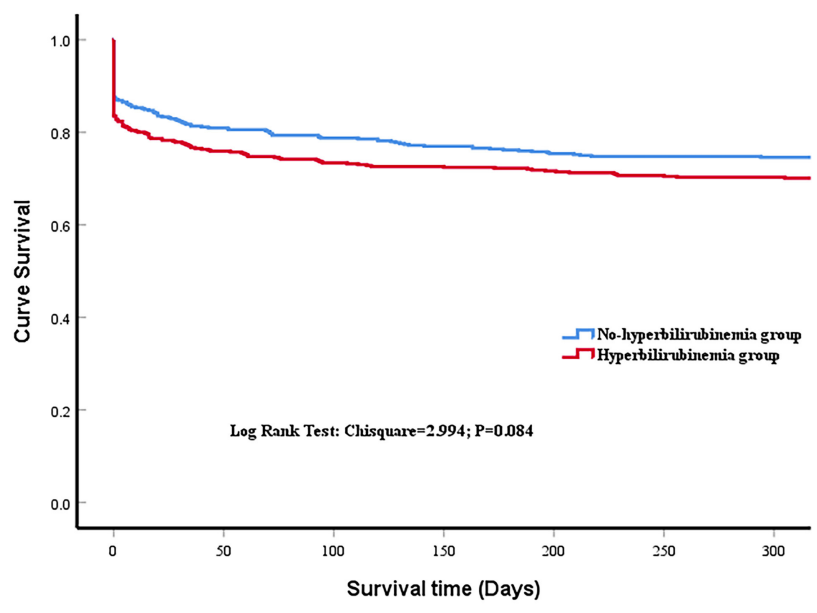

D

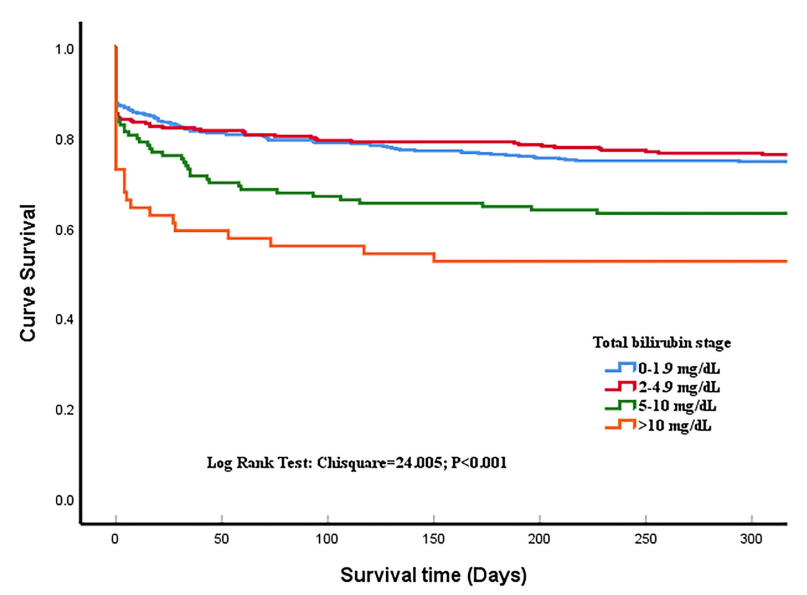

FIGURE 2 | Time to death analysis. (A) shows the survival curves for all included patients at 1 year after admission of the last patients before matching, with a significant difference between liver injury and non-liver injury groups (Log-rank $P=0.006$ ). (B) shows the survival curves for different stages of serum total bilirubin (TBIL) levels before propensity matching. Kaplan-Meier analysis showed survival time did not differ between the hyperbilirubinemia group and the normal group. Log-rank $p<0.001$. (C) shows the survival curves between liver injury and non-liver injury groups after matching (log-rank $P=0.084)$; (D) shows the survival curves for different stages of serum TBIL levels after propensity matching (Log-rank $p<0.001)$.

ventilation [HR 1.281; 95\% CI (1.093-1.501); $P=0.002]$ were independent risk factors in 1-year mortality before matching by stepwise multivariate Cox hazard analysis.

\section{PS Analysis}

From the above results, we found that the baseline clinical information significantly influenced the hyperbilirubinemia group and long-term clinical outcomes. Thus, we applied propensity-score matching to minimize confounding biases. One-to-one propensity-score matching yielded a cohort of 532 patients in the hyperbilirubinemia group and 532 in the control group. Baseline characteristics for patients with and without hyperbilirubinemia were well-balanced after matching, as shown in Table 4. Standardized biases for all variables were.05 or less. The SOFA score at admission was excluded as a matching variable for one of its evaluated parameters, including TBIL. In the hyperbilirubinemia group after matching, the rates of the SOFA score at admission above 4 was significantly higher in the hyperbilirubinemia group when compared with the non-hyperbilirubinemia group (83.4 vs. $59.8 \% ; p<0.001)$.

The matched results also showed that the patients with sepsis with the new onset of hyperbilirubinemia were associated with significantly increased risks of mortality at the hospital (16. vs. $11.8 \%, P=0.036)$, 30 days ( 21.4 vs. $16.7 \%, P=0.038)$, 90 days (25 vs. $19.5 \%, P=0.027$ ), and 1 year ( 29.5 vs. $24.4 \%, P=0.047)$, but not statistically increased in 90 days, 180 days, and 1-year mortality in the overall population (Table 5). The duration of mechanical ventilation, vasoactive drug or sedative drug usage, and AKI stage did not significantly differ between the two groups. 
TABLE 3 | Univariate and stepwise multivariate Cox hazard analysis of risk factors for mortality in sepsis before matching.

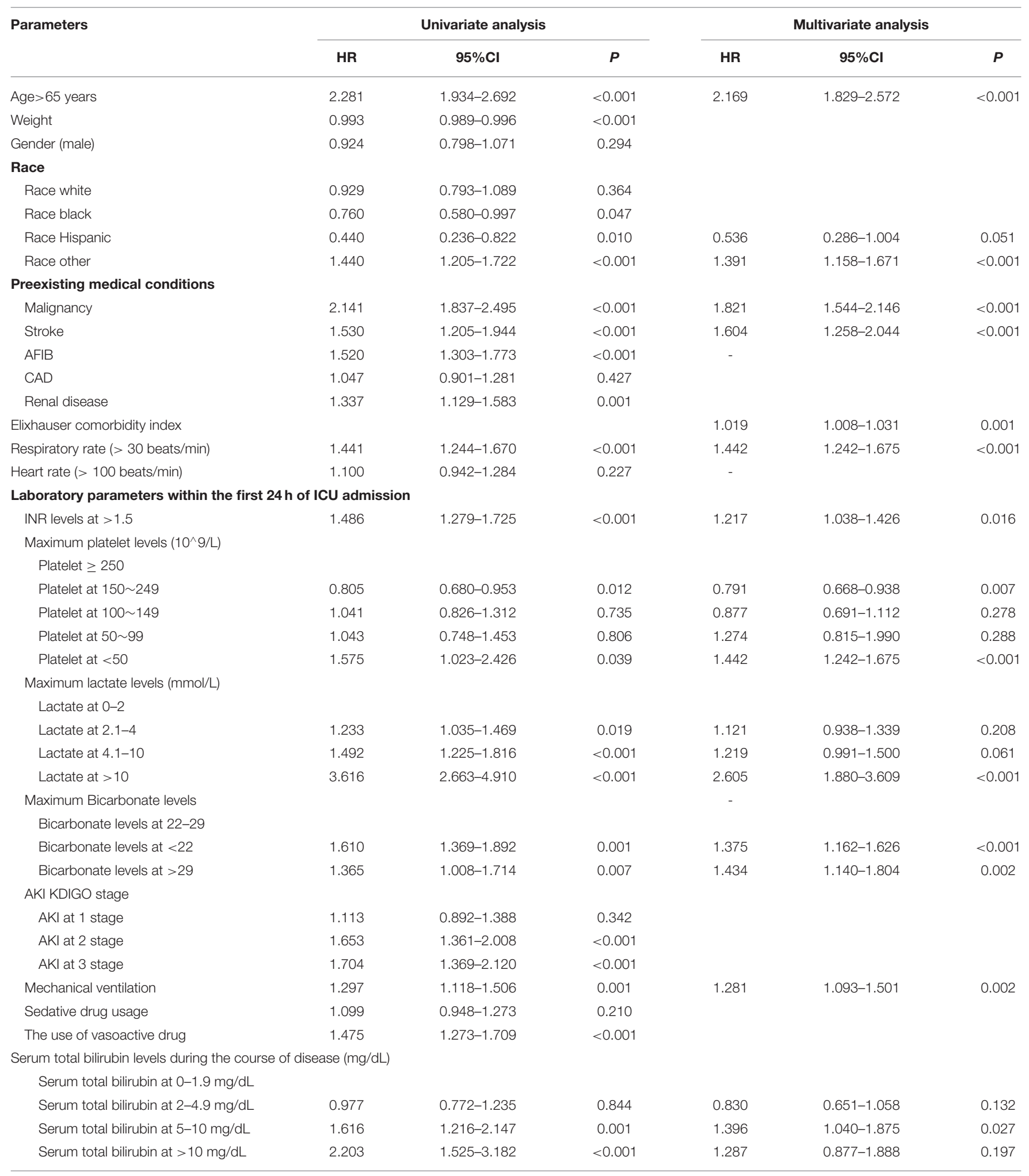

AFIB atrial fibrillation, INR International Normalized Ratio, BUN blood urea nitrogen, KDIGO the Kidney Disease: Improving Global Outcomes.

After matching, Kaplan-Meier's analysis showed that non-hyperbilirubinemia group but did not reach statistical the patients with new onset hyperbilirubinemia had significance $(P=0.084 ;$ Figure 2C). However, TBIL a lower long-term survival rate compared with the levels more than or equal to $5 \mathrm{mg} / \mathrm{dl}$ at the hospital also 
TABLE 4 | Demographic characteristics and clinical outcomes for hyperbilirubinemia and non-hyperbilirubinemia groups after propensity score matching.

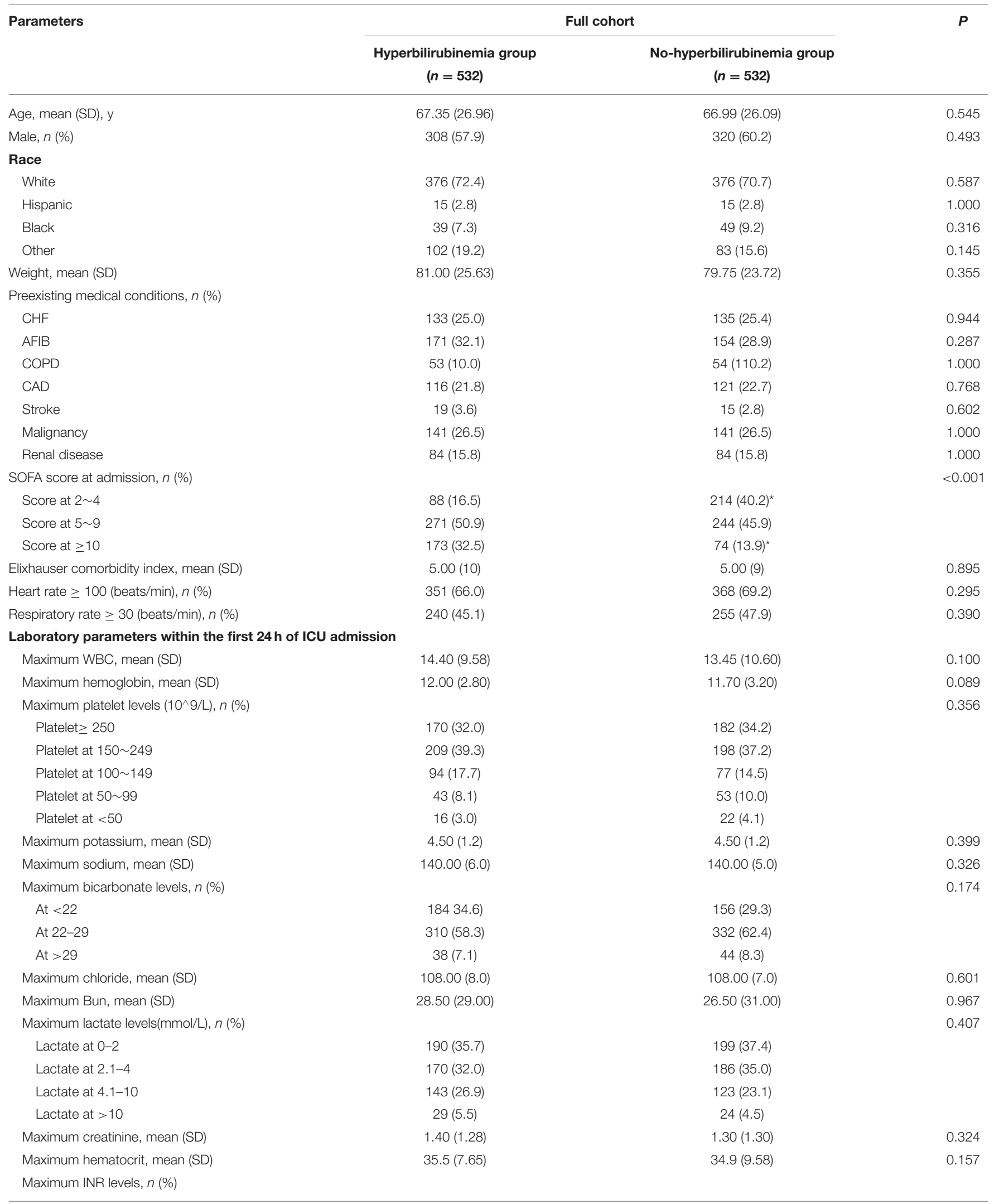


TABLE 4 | Continued

\begin{tabular}{|c|c|c|c|}
\hline Parameters & \multicolumn{2}{|c|}{ Full cohort } & $P$ \\
\hline At $>1.5$ & $276(51.9)$ & $270(50.8)$ & 0.759 \\
\hline Maximum PH, mean (SD) & $7.40(0.07)$ & $7.40(0.07)$ & 0.796 \\
\hline Maximum pO2, mean (SD) & $214.0(111)$ & $214.0(144)$ & 0.501 \\
\hline Maximum ALT, mean (SD) & $115.00(288.00)$ & $33.50(70.75)$ & $<0.001$ \\
\hline Maximum AST, mean (SD) & $152.50(354.50)$ & $43.00(120.50)$ & $<0.001$ \\
\hline Maximum total bilirubin at $24 \mathrm{~h}$, mean (SD) & $3.30(3.10)$ & $0.60(0.60)$ & $<0.001$ \\
\hline Maximum total bilirubin at $48 \mathrm{~h}$, mean (SD) & $2.10(3.20)$ & $0.00(0.50)$ & $<0.001$ \\
\hline
\end{tabular}

Continuous variables are reported as mean (Standard deviation, SD), and Categorical variables are reported as count (\% of column total).

CHF congestive heart failure, AFIB atrial fibrillation, CAD coronary artery disease, COPD chronic obstructive pulmonary disease, SOFA Sequential Organ Failure Assessment, WBC white blood cell, INR International Normalized Ratio, BUN blood urea nitrogen, AST aspartate transaminase, ALT alanine transaminase, pCO2 partial pressure of carbon dioxide, pO2 partial pressure of oxygen.

*Represent significant difference with $P<0.05$.

TABLE 5 | Clinical outcomes between hyperbilirubinemia and non-hyperbilirubinemia groups after propensity score matching.

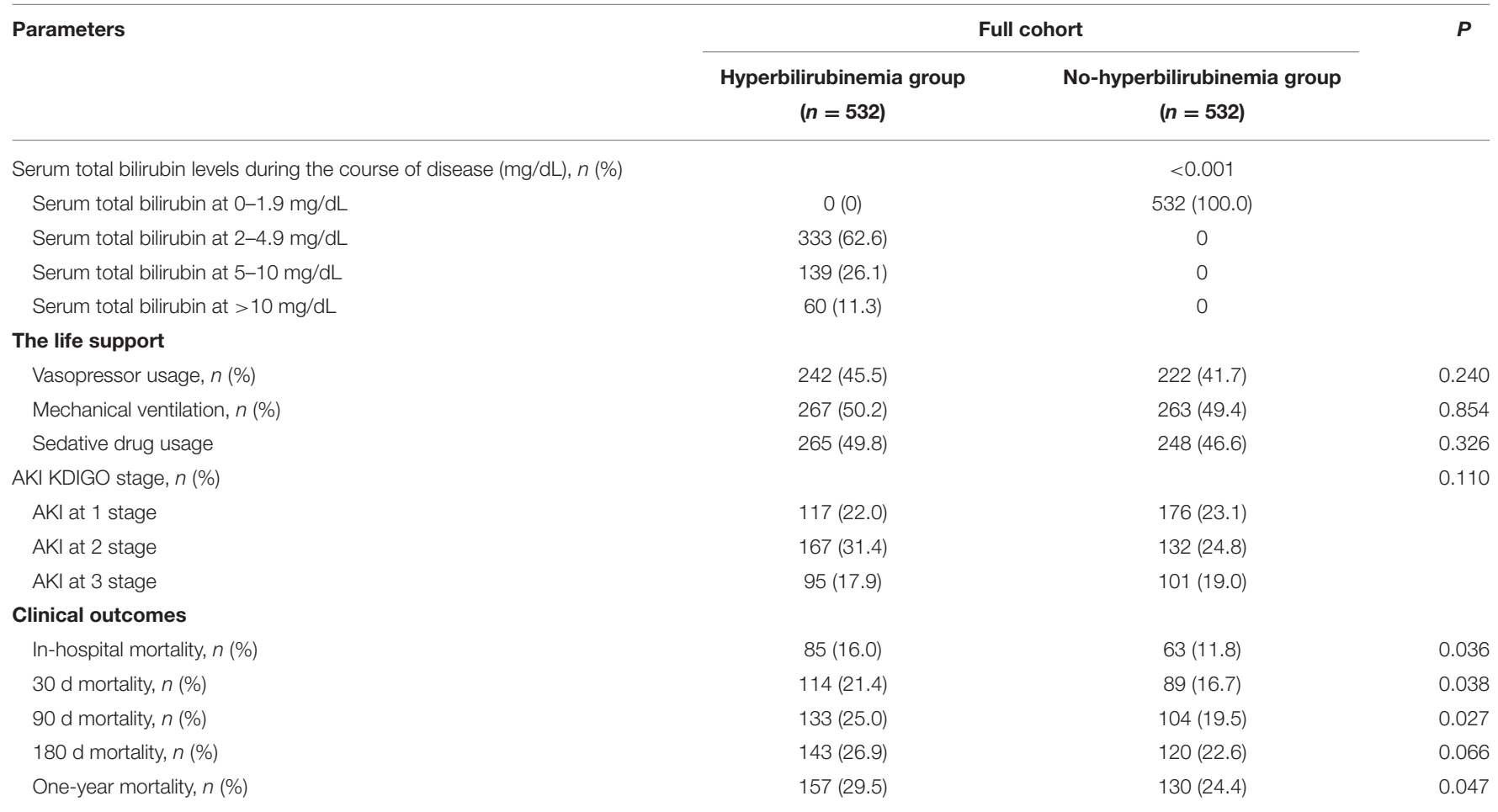

Categorical variables are reported as count (\% of column total).

AKI acute kidney injury, KDIGO the Kidney Disease: Improving Global Outcomes.

significantly increases the risk of mortality in sepsis $(p<0.001$, Figure 2D).

To further verify the levels of TBIL at the hospital on clinical outcomes, we then classified the patients into TBIL levels at the $<5 \mathrm{mg} / \mathrm{dl}$ group and TBIL levels at the $\geq 5 \mathrm{mg} / \mathrm{dl}$ group. The results demonstrated that patients with sepsis with serum
TBIL levels at $\geq 5 \mathrm{mg} / \mathrm{dl}$ during the hospital stage could bring significant poor outcomes on 30-day (28.1 vs. $17 \%)$, 180-day (34.7 vs. $19.4 \%$ ), and 1 -year mortality (37.7 vs. $21.7 \%$ ), but with noninfluence on hospital mortality (18.6 vs. $12.8 \%$ ) when compared with those with TBIL levels at the $<5 \mathrm{mg} / \mathrm{dl}$ group (Supplementary Table 1). 
TABLE 6 | Univariate and multivariate regression analysis to explore independent predictors affecting long-term mortality in sepsis after propensity score matching.

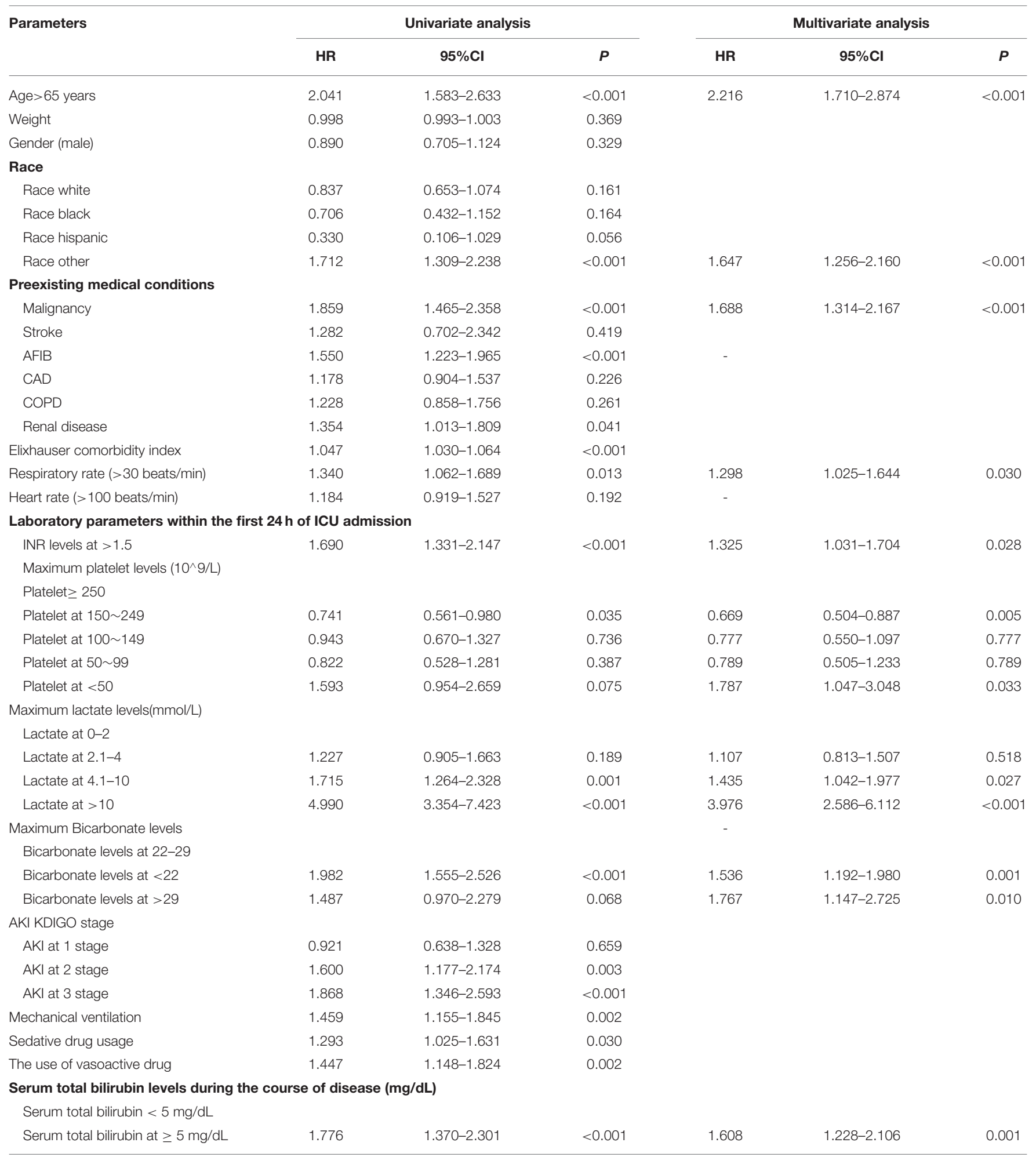

CHF congestive heart failure, AFIB atrial fibrillation, CAD coronary artery disease, COPD chronic obstructive pulmonary disease, SOFA Sequential Organ Failure Assessment. AKI acute kidney injury, KDIGO the Kidney Disease: Improving Global Outcomes. 


\section{Univariate and Multivariate Cox Hazard Proportional Analysis to Explore Independent Predictors Affecting Long-Term Mortality in Sepsis After Matching}

We then explored the direct effects of different levels of hyperbilirubinemia during the cause of sepsis on a long-term clinical outcome after matching. Multivariate analysis using the Cox proportional hazard model for all variables was identified as significant by univariate analysis. Following control of confounders and LR forward elimination, it was still shown in Table 6 that, when TBIL levels were $<5 \mathrm{mg} / \mathrm{dl}$ as a reference variable, TBIL at $\geq 5 \mathrm{mg} / \mathrm{dl}$ during sepsis increased the risk of 1-year mortality with 1.608 times [95\% CI $(1.228-2.106)$; $P=0.001]$.

We also found that age above 65 years old [HR 2.216; $95 \%$ CI (1.710-2.874); $p<0.001$ ], other race [HR 1.647; 95\% CI $(1.256-2.160) ; p<0.001$ ], preexisting malignancy [HR 1.688; 95\% CI (1.314-2.167); $p<0.001]$, respiratory rate above 30 beats/min at admission [HR 1.298; 95\% CI (1.025-1.644); $P=$ 0.030 ], serum INR above 1.5 at $24 \mathrm{~h}$ of admission [HR 1.325; 95\% CI (1.031-1.704); $P=0.028]$, serum platelet $<50^{*} 10^{\wedge} 9 / \mathrm{L}$ at $24 \mathrm{~h}$ of admission [HR 1.787; 95\% CI (1.047-3.048); $P=$ 0.033 ], serum lactate more than $4 \mathrm{mmol} / \mathrm{L}$ [HR $1.435 ; 95 \%$ CI (1.042-1.977); $P=0.027]$, or even more than $10 \mathrm{mmol} / \mathrm{L}$ at $24 \mathrm{~h}$ of admission [HR 3.976; 95\% CI (2.586-6.112); $p<0.001$ ], and serum bicarbonate $<22 \mathrm{mmol} / \mathrm{L}$ [HR 1.536; 95\% CI (1.1921.980); $P=0.001$ ], or above $29 \mathrm{mmol} / \mathrm{L}$ [HR 1.767; 95\% CI $(1.147-2.725) ; P=0.010]$ were independent risk factors in 1-year mortality in sepsis after matchings. When using serum platelet $\geq 250^{*} 10^{\wedge} 9 / \mathrm{L}$ as a reference variable, the result demonstrated that serum platelet at $150-249^{*} 10^{\wedge} 9 / \mathrm{L}$ was the only protective factor in long-term mortality in sepsis [HR 0.669; 95\% CI $(0.504-0.887 ; P=0.005)]$.

\section{DISCUSSION}

In this large cohort study, we find the incidence rate of the new onset of hyperbilirubinemia during sepsis is $19.5 \%$, which is considerably lower than the rate of hyperbilirubinemia in other patients who are critically ill $(14,27)$. The difference is mainly due to the hyperbilirubinemia in our study that is defined as the new onset excluding past liver diseases, while previous studies included patients with preexisting chronic liver conditions $(22,28)$. Our data show that, after PS matching, the new onset of hyperbilirubinemia during sepsis cannot significantly increase the risk of long-term mortality, but the patients with TBIL levels are more than or equal to $5 \mathrm{mg} / \mathrm{dl}$ do. Further multivariate Cox hazard analysis shows that age above 65 years old, other race, preexisting malignancy, respiratory rate above 30 beats/min at admission, serum parameters levels within 24 -h admission containing INR above 1.5 , platelet $<50^{*} 10^{\wedge} 9 / \mathrm{L}$, lactate above $4 \mathrm{mmol} / \mathrm{L}$, bicarbonate $<22$ or above $29 \mathrm{mmol} / \mathrm{L}$, serum TBIL during sepsis at more than or equal to $5 \mathrm{mg} / \mathrm{dl}$ are the independent risk factors in 1-year mortality of patients with sepsis.

Furthermore, the low level of platelet, the INR level more than 1.5 , the respiratory rate more than or equal to 30 (beats $/ \mathrm{min}$ ), the higher creatinine level, the higher arterial $\mathrm{pCO}$, and the lower bicarbonate level in the hyperbilirubinemia group compared with the non-hyperbilirubinemia group indicate that there is a close correlation between hyperbilirubinemia and the deterioration of hematologic, coagulation, respiratory, urinary, and acid-base balance systems function in sepsis. In stepwise multivariate Cox hazard analysis, Elixhauser comorbidity index, the respiratory rate above 30 beats/min at admission, serum INR above 1.5, serum platelet $<50^{*} 10^{\wedge} 9 / \mathrm{L}$, serum lactate more than $4 \mathrm{mmol} / \mathrm{L}$, serum bicarbonate $<22 \mathrm{mmol} / \mathrm{L}$, or above $29 \mathrm{mmol} / \mathrm{L}$ at $24 \mathrm{~h}$ of admission, and mechanical ventilation as independent risk factors in 1-year mortality of patients with sepsis hint that hematologic, coagulation, respiratory, and acid-base systems that are imbalance at the early stage of sepsis predict poor prognosis of long-term mortality of sepsis. In addition, when serum TBIL at more than or equal to $5 \mathrm{mg} / \mathrm{dl}$ as a predictive factor in 1-year mortality of patients with sepsis consolidates our speculation: the new onset of hyperbilirubinemia reach at some extent aggravates sepsis prognosis.

Intriguingly, we observe that the hyperbilirubinemia group has a significantly higher rate of preexisting AFIB and malignancy but a lower rate of past COPD, stroke, and renal disease than the non-hyperbilirubinemia group. The mechanism of the potential relationship between the new onset of hyperbilirubinemia and past medical history in sepsis is not clear and needs further study. Multivariate Cox analysis results suggest that preexisting diseases, such as malignancy, affect the long-term survival of patients with sepsis, which is consistent with recent findings (29). Given that great bias was frequently generated when analyzing the sole effect of hyperbilirubinemia on sepsis, we used PS matching analysis to balance baseline characteristics to minimize confounding bias. After matching, baseline variants are well adjusted to parallel, thus reducing confounding bias to the maximum extent. Importantly, the new onset of hyperbilirubinemia when TBIL levels achieve at more than or equal to $5 \mathrm{mg} / \mathrm{dl}$ is associated with significantly increased long-term mortality of patients with sepsis after matching. Compared with the previous study, which did not use PS matching to strictly control possible confounders (24), our study provides solid evidence and comprehensive dissection of the relationship between the dynamic change of hyperbilirubinemia and sepsis prognosis, and, at the same time, we seek a cutoff value of TBIL levels associated with a poor outcome in sepsis, which may guide for early intervention for ICU physicians. Our results are consistent with previous basic research, which shows that the liver plays an important role in endotoxin-induced acute lung injury (30). Moreover, Zhang et al. (31) have proposed a Cox regression model with time-varying covariates, which is useful for evaluating the dynamic change of hyperbilirubinemia on clinical outcomes and can be used in further analysis. The liver is the key detoxification organ and the critical site to clear invasive pathogens and alleviate inflammation reaction by exerting innate immune system function $(6,7)$. Meanwhile, liver dysfunction 
and associated hyperbilirubinemia lead to inflammation and immune response out of control and cascading organ damage in sepsis $(8,9)$.

Furthermore, we explore the independent risk factors affecting 1-year mortality of patients with sepsis after PS matching, and we find that old age, preexisting malignancy, respiratory rate above 30 beats/min at admission, serum parameters within 24 $\mathrm{h}$ admission with INR above 1.5 , serum platelet $<50^{*} 10^{\wedge} 9 / \mathrm{L}$, serum lactate more than $4 \mathrm{mmol} / \mathrm{L}$, and bicarbonate $<22$ or above $29 \mathrm{mmol} / \mathrm{L}$ are the independent risk factors in 1year mortality of patients with sepsis. These parameters may provide early warning of the prognosis of patients with sepsis. Previous basic pieces of research show that sepsis-induced acute kidney and myocardial injury are age-dependent $(32,33)$, and a recent clinical study has shown that the odds for mortality of patients with sepsis in ICU increase with age (34), which are consistent with our results. In qSOFA definition, expert consensus shows the respiratory rate of $22 / \mathrm{min}$ or greater, which is the predictor for a poor outcome of patients with sepsis (25); our results further demonstrate the respiratory rate of $30 / \mathrm{min}$ or greater at admission is the independent risk factor in long-term mortality. Abundant studies have focused on the correlation between the serum lactate level and shortterm mortality of patients with sepsis; the lactate level $\geq 2$ $\mathrm{mmol} / \mathrm{L}$ around is demonstrated having the predictive value $(35,36)$. However, the cutoff of the lactate level for predicting long-term mortality of sepsis remains unclear. Our results show lactate above $4 \mathrm{mmol} / \mathrm{L}$ within 24 -h admission is the independent predictor for 1-year mortality of sepsis, and patients with lactate above $10 \mathrm{mmol} / \mathrm{L}$ will bring a poor prognosis in sepsis. Of note, recent studies have shown that platelets play a vital role in immunological surveillance against pathogens invaders and contribute to innate immune system function (37, 38). Wong et al. demonstrated that platelets collaborate with macrophages to fight against certain blood-borne infections. In addition, the absence of the platelet resulted in the platelet being unable to localize to the sites of infection, leading to rapid death of the Kupffer cells and endothelium, followed by more leakage of plasma out of blood vessels, and even host mortality (39). This may explain why patients with sepsis with lower-level platelets have poor outcomes in our study. Besides, we find that bipolar serum bicarbonate levels are another risk factor in the long-term mortality of patients with sepsis. Previous experimental studies indicate that endotoxemia significantly decreases bile acid-independent bile flow (BAIBF) and associated biliary $\mathrm{HCO}_{3}^{-}$output (40), thus reducing the serum bicarbonate level. As there are no studies that have reported the correlation between the bicarbonate level and sepsis mortality, our study first shows that bicarbonate $<22$ or above $29 \mathrm{mmol} / \mathrm{L}$ is an independent predictor for 1-year mortality of sepsis.

To our knowledge, this is the first and largest study so far to dissect the correlation between the dynamic change of hyperbilirubinemia and outcomes of sepsis. However, there are several limitations to our study. First, we used the database from a single academic medical center in the USA; therefore, some of the cases from almost 10 years ago, as diagnosis or treatment strategies at that time, would be inconsistent with current guidelines, which brings great bias. There is residual confounding by variables not collected into the MIMIC-III database. However, we include all the patients with sepsis according to the uniform standard of Sepsis 3.0 and apply PSs analysis and match the baseline characteristics of the patients to eliminate confounding factors and decrease bias to the maximum. Second, there are a few missing data that bring bias, yet we delete the data whose missing percentage is larger than $50 \%$ to decrease bias. The meanvalue imputation algorithm is selected to substitute missing values. Third, the single-centered design restricts generalizability to apply our conclusion to other regions, while we use a large sample size and PS matching analysis to guarantee the quality of our study.

\section{CONCLUSION}

In conclusion, patients with serum TBIL at more than or equal to $5 \mathrm{mg} / \mathrm{dl}$ during sepsis decrease survival rates after PS matching. In addition, we conclude that age above 65 years old, preexisting malignancy, respiratory rate above 30 beats/min at admission, serum parameter levels within 24 -h admission, containing INR above 1.5 , platelet $<50^{*} 10^{\wedge} 9 / \mathrm{L}$, lactate above $4 \mathrm{mmol} / \mathrm{L}$, and bicarbonate $<22$ or above $29 \mathrm{mmol} / \mathrm{L}$ are independent risk predictors for long-term mortality of sepsis. Our study provides solid evidence and will rekindle the awareness of the risk factors leading to poor prognosis in sepsis.

\section{DATA AVAILABILITY STATEMENT}

The original contributions presented in the study are included in the article/Supplementary Files, further inquiries can be directed to the corresponding authors.

\section{ETHICS STATEMENT}

The Ethics approval statement was approved by the Institutional Review Boards of Beth Israel Deaconess Medical Center (Boston, $\mathrm{MA}$ ), and all the data were under a deidentification process to protect individual privacy and waived the need for a patient consent statement. Written informed consent from the participants' legal guardian/next of kin was not required to participate in this study in accordance with the national legislation and the institutional requirements.

\section{AUTHOR CONTRIBUTIONS}

MP, DQ, LZ, and ZH contributed to the conception and design of the research. MP and FD contributed to the acquisition and analysis of the data. MP, $\mathrm{ZH}$, and $\mathrm{LZ}$ contributed to the interpretation of the results. MP and DQ drafted the manuscript. $\mathrm{DQ}, \mathrm{ZH}$, and $\mathrm{LZ}$ revised the manuscript. All authors have agreed to be fully accountable for ensuring the accuracy of the work, and approved the final manuscript.

\section{FUNDING}

This work was supported by Changsha Municipal Natural Science Foundation (grant number: kq2007078). 


\section{ACKNOWLEDGMENTS}

We are grateful to Professor Liu WW for his kind help in the consultation of statistical problems.

\section{REFERENCES}

1. Reinhart K, Daniels R, Kissoon N, Machado FR, Schachter RD, Finfer S. Recognizing sepsis as a global health priority-a WHO resolution. $N$ Engl J Med. (2017) 377:414-7. doi: 10.1056/NEJMp 1707170

2. Cecconi M, Evans L, Levy M, Rhodes A. Sepsis and septic shock. Lancet. (2018) 392:75-87. doi: 10.1016/S0140-6736(18) 30696-2

3. Wong HR. Pediatric sepsis biomarkers for prognostic and predictive enrichment. Pediatr Res. (2021) doi: 10.1038/s41390-02101620-5

4. Zhang K, Zhang S, Cui W, Hong Y, Zhang G, Zhang Z. Development and validation of a sepsis mortality risk score for sepsis-3 patients in intensive care unit. Front Med (Lausanne). (2020) 7:609769. doi: 10.3389/fmed.2020. 609769

5. Zhang Z, Zhang G, Goyal H, Mo L, Hong Y. Identification of subclasses of sepsis that showed different clinical outcomes and responses to amount of fluid resuscitation: a latent profile analysis. Crit Care. (2018) 22:347. doi: 10.1186/s13054-018-2279-3

6. Yan J, Li S, Li S. The role of the liver in sepsis. Int Rev Immunol. (2014) 33:498-510. doi: 10.3109/08830185.2014.889129

7. Jenne CN, Kubes P. Immune surveillance by the liver. Nat Immunol. (2013) 14:996-1006. doi: 10.1038/ni.2691

8. Bauer M, Press AT, Trauner M. The liver in sepsis: patterns of response and injury. Curr Opin Crit Care. (2013) 19:123-7. doi: 10.1097/MCC.0b013e32835eba6d

9. Strnad P, Tacke F, Koch A, Trautwein C. Liver - guardian, modifier and target of sepsis. Nat Rev Gastroenterol Hepatol. (2017) 14:55-66. doi: $10.1038 /$ nrgastro. 2016.168

10. Fuchs M, Sanyal AJ. Sepsis and cholestasis. Clin Liver Dis. (2008) 12:151-72, ix. doi: $10.1016 /$ j.cld.2007.11.002

11. Iwatani S, Jacobsen DR, Wong RJ, Stevenson DK. Low-dose lipopolysaccharide exposure can increase in vivo bilirubin production rates in newborn mice. Acta Paediatr. (2019) 109:1551-9 doi: 10.1111/apa. 15143

12. Spapen H. Liver perfusion in sepsis, septic shock, and multiorgan failure. Anat Rec (Hoboken). (2008) 291:714-20. doi: 10.1002/ar.20646

13. Jager B, Drolz A, Michl B, Schellongowski P, Bojic A, Nikfardjam $\mathrm{M}$, et al. Jaundice increases the rate of complications and one-year mortality in patients with hypoxic hepatitis. Hepatology. (2012) 56:2297-304. doi: 10.1002/hep.25896

14. te Boekhorst T, Urlus M, Doesburg W, Yap SH, Goris RJ. Etiologic factors of jaundice in severely ill patients. A retrospective study in patients admitted to an intensive care unit with severe trauma or with septic intraabdominal complications following surgery and without evidence of bile duct obstruction. J Hepatol. (1988) 7:111-7. doi: 10.1016/S0168-8278(88) 80514-2

15. Koskinas J, Gomatos IP, Tiniakos DG, Memos N, Boutsikou M, Garatzioti A, et al. Liver histology in ICU patients dying from sepsis: a clinico-pathological study. World J Gastroenterol. (2008) 14:1389-93. doi: 10.3748/wjg. 14.1389

16. Trauner M, Meier PJ, Boyer JL. Molecular pathogenesis of cholestasis. N Engl J Med. (1998) 339:1217-27. doi: 10.1056/NEJM1998102233 91707

17. Kramer L, Jordan B, Druml W, Bauer P, Metnitz PG, Austrian Epidemiologic Study on Intensive Care ASG. Incidence and prognosis of early hepatic

\section{SUPPLEMENTARY MATERIAL}

The Supplementary Material for this article can be found online at: https:/www.frontiersin.org/articles/10.3389/fmed. 2021.713917/full\#supplementary-material

dysfunction in critically ill patients-a prospective multicenter study. Crit Care Med. (2007) 35:1099-104. doi: 10.1097/01.CCM.0000259462.97164.A0

18. Chen J, Yu JL. [Predictive factors for poor prognosis of neonates with early-onset sepsis]. Zhongguo Dang Dai Er Ke Za Zhi. (2020) 22:146-51. doi: 10.7499/j.issn.1008-8830.2020.02.012

19. DA S, L PS, A GdM, O G, Hepatology KPJ. Management of sepsis in patients with cirrhosis: current evidence and practical approach. Hepatology. (2019) 70:418-28. doi: 10.1002/hep.30412

20. Jenniskens M, Langouche L, Van den Berghe G. Cholestatic alterations in the critically ill: some new light on an old problem. Chest. (2018) 153:733-43. doi: 10.1016/j.chest.2017.08.018

21. Brun-Buisson C, Meshaka P, Pinton P, Vallet B, Group ES. EPISEPSIS: a reappraisal of the epidemiology and outcome of severe sepsis in French intensive care units. Intensive Care Med. (2004) 30:580-8. doi: 10.1007/s00134-003-2121-4

22. Zhai R, Sheu CC, Su L, Gong MN, Tejera P, Chen F, et al. Serum bilirubin levels on ICU admission are associated with ARDS development and mortality in sepsis. Thorax. (2009) 64:784-90. doi: 10.1136/thx.2009. 113464

23. Vincent JL, Angus DC, Artigas A, Kalil A, Basson BR, Jamal HH, et al. Effects of drotrecogin alfa (activated) on organ dysfunction in the PROWESS trial. Crit Care Med. (2003) 31:834-40. doi: 10.1097/01.CCM.0000051515. 56179.E1

24. Nesseler N, Launey Y, Aninat C, White J, Corlu A, Pieper K, et al. Liver dysfunction is associated with long-term mortality in septic shock. Am J Respir Crit Care Med. (2016) 193:335-7. doi: 10.1164/rccm.2015081660LE

25. Singer M, Deutschman CS, Seymour CW, Shankar-Hari M, Annane D, Bauer $\mathrm{M}$, et al. The third international consensus definitions for sepsis and septic shock (Sepsis-3). JAMA. (2016) 315:801-10. doi: 10.1001/jama.2016.0287

26. Vincent JL, Moreno R, Takala J, Willatts S, De Mendonca A, Bruining H, et al. The SOFA (Sepsis-related Organ Failure Assessment) score to describe organ dysfunction/failure. On behalf of the Working Group on Sepsis-Related Problems of the European Society of Intensive Care Medicine. Intensive Care Med. (1996) 22:707-10. doi: 10.1007/BF01709751

27. Brienza N, Dalfino L, Cinnella G, Diele C, Bruno F, Fiore T. Jaundice in critical illness: promoting factors of a concealed reality. Intensive Care Med. (2006) 32:267-74. doi: 10.1007/s00134-005-0023-3

28. Franson TR, Hierholzer WJ, Jr., LaBrecque DR. Frequency and characteristics of hyperbilirubinemia associated with bacteremia. Rev Infect Dis. (1985) 7:1-9. doi: 10.1093/clinids/7.1.1

29. Dimopoulos G, Rovina N, Patrani M, Antoniadou E, Konstantonis D, Vryza K, et al. Past history of stage I/II solid tumor malignancy impacts considerably on sepsis mortality: a propensity score matching analysis from the hellenic sepsis study group. BMC Infect Dis. (2019) 19:831. doi: 10.1186/s12879-019-4448-7

30. Siore AM, Parker RE, Stecenko AA, Cuppels C, McKean M, Christman BW, et al. Endotoxin-induced acute lung injury requires interaction with the liver. Am J Physiol Lung Cell Mol Physiol. (2005) 289:L769-76. doi: 10.1152/ajplung.00137.2005

31. Zhang Y, Li D, Yang Y, Su J, Xu X, Wang M, et al. Clinical and molecular characteristics of Chryseobacterium indologenes isolates at a teaching hospital in Shanghai, China. Ann Transl Med. (2021) 9:668. doi: 10.21037/atm-21-933

32. Maddens B, Vandendriessche B, Demon D, Vanholder R, Chiers K, Cauwels A, et al. Severity of sepsis-induced acute kidney injury in a novel mouse model is age dependent. Crit Care Med. (2012) 40:2638-46. doi: 10.1097/CCM.0b013e3182591ebe 
33. Checchia PA, Schierding W, Polpitiya A, Dixon D, Macmillan S, Muenzer J, et al. Myocardial transcriptional profiles in a murine model of sepsis: evidence for the importance of age. Pediatr Crit Care Med. (2008) 9:530-5. doi: 10.1097/PCC.0b013e3181849a2f

34. Kotfis K, Wittebole X, Jaschinski U, Sole-Violan J, Kashyap R, Leone M, et al. A worldwide perspective of sepsis epidemiology and survival according to age: Observational data from the ICON audit. J Crit Care. (2019) 51:122-32. doi: 10.1016/j.jcrc.2019.02.015

35. Ryoo SM, Lee J, Lee YS, Lee JH, Lim KS, Huh JW, et al. Lactate level versus lactate clearance for predicting mortality in patients with septic shock defined by Sepsis-3. Crit Care Med. (2018) 46:e489-95. doi: 10.1097/CCM.0000000000003030

36. Filho RR, Rocha LL, Correa TD, Pessoa CM, Colombo G, Assuncao MS. Blood lactate levels cutoff and mortality prediction in sepsis-time for a reappraisal? a Retrospective Cohort Study. Shock. (2016) 46:480-5. doi: 10.1097/SHK.0000000000000667

37. Surewaard BGJ, Thanabalasuriar A, Zeng Z, Tkaczyk C, Cohen TS, Bardoel BW, et al. alpha-toxin induces platelet aggregation and liver injury during Staphylococcus aureus sepsis. Cell Host Microbe. (2018) 24:271-84 e3. doi: 10.1016/j.chom.2018.06.017

38. Broadley SP, Plaumann A, Coletti R, Lehmann C, Wanisch A, Seidlmeier A, et al. Dual-Track clearance of circulating bacteria balances rapid restoration of blood sterility with induction of adaptive immunity. Cell Host Microbe. (2016) 20:36-48. doi: 10.1016/j.chom.2016.05.023

39. Wong CH, Jenne CN, Petri B, Chrobok NL, Kubes P. Nucleation of platelets with blood-borne pathogens on Kupffer cells precedes other innate immunity and contributes to bacterial clearance. Nat Immunol. (2013) 14:785-92. doi: 10.1038/ni.2631

40. Trauner M, Nathanson MH, Rydberg SA, Koeppel TA, Gartung C, Sessa WC, et al. Endotoxin impairs biliary glutathione and HCO3- excretion and blocks the choleretic effect of nitric oxide in rat liver. Hepatology. (1997) 25:1184-91. doi: 10.1002/hep.510250522

Conflict of Interest: The authors declare that the research was conducted in the absence of any commercial or financial relationships that could be construed as a potential conflict of interest.

Publisher's Note: All claims expressed in this article are solely those of the authors and do not necessarily represent those of their affiliated organizations, or those of the publisher, the editors and the reviewers. Any product that may be evaluated in this article, or claim that may be made by its manufacturer, is not guaranteed or endorsed by the publisher.

Copyright (C) 2021 Peng, Deng, Qi, Hu and Zhang. This is an open-access article distributed under the terms of the Creative Commons Attribution License (CC BY). The use, distribution or reproduction in other forums is permitted, provided the original author(s) and the copyright owner(s) are credited and that the original publication in this journal is cited, in accordance with accepted academic practice. No use, distribution or reproduction is permitted which does not comply with these terms. 